\title{
GESTÃO DA FORÇA DE TRABALHO E PLANEJAMENTO DE FÉRIAS: UM ESTUDO DE CASO EM AGÊNCIAS BANCÁRIAS
}

\author{
Marco Antonio Bonelli Junior \\ Universidade Estadual de Campinas - UNICAMP \\ R. Pedro Zaccaria, 1300, Limeira - SP \\ m228351@dac.unicamp.br \\ Cleber Damião Rocco \\ Universidade Estadual de Campinas - UNICAMP \\ R. Pedro Zaccaria, 1300, Limeira - SP \\ cdrocco@unicamp.br \\ Andre Luiz Barbosa Lima \\ Universidade Federal de Ouro Preto - UFOP \\ R. 36, 115, João Monlevade - MG \\ andre.lima1@aluno.ufop.edu.br
}

\section{RESUMO}

O problema de alocação de férias e força de trabalho tem sido um campo amplo para a a realização de pesquisas nas áreas de gestão e pesquisa operacional. Entretanto, mesmo sendo um problema comum e conhecido, encontrar boas soluções é normalmente uma tarefa difıcil dado à sua caracterıstica combinatorial, sendo ainda mais dificil encontrar a solução ótima de forma a minimizar os custos, adequar o planejamento às preferências dos trabalhadores, distribuir os turnos de forma igualitária e satisfazer às restrições de necessidade de trabalho. Este artigo trata do problema de alocação de férias e força de trabalho considerando um cenário do setor bancário, desenvolvendo uma abordagem de solução baseada em procedimentos heurísticos e de programação linear inteira mista. Como resultado, a abordagem proposta foi capaz de gerar uma matriz de cobertura e alocação para os trabalhadores e identificar insuficiências na capacidade de cobertura de férias da empresa.

Palavras-chave: planejamento da força de trabalho; agências bancárias; modelagem matemática; otimização. 


\begin{abstract}
The problem of vacation and workforce allocation has been a broad field for conducting research in management and operational research. However, even though it is a common and well-known problem, finding good solutions is usually a difficult task given its combinatorial feature, making it even more difficult to find the optimal solution in order to minimize costs, tailor planning to workers' preferences, distribute shifts. equally and meet the constraints of labor This paper addresses the vacation and workforce allocation problem considering a banking sector scenario, developing a solution approach based on heuristic procedures and mixed integer linear programming. As a result, the proposed approach was able to generate a coverage and allocation matrix for workers and identify shortcomings in the company's vacation coverage capacity.
\end{abstract}

Keywords: workforce planning; bank branches; mathematical modelling; optimization.

\title{
Como Citar:
}

BONELli JUNIOR, M. A.; ROCCO, C. D.; LIMA, A. L. B. Gestão da Força de Trabalho e Planejamento de Férias: Um Estudo de Caso em Agências Bancárias. In: SIMPÓSIO DE PESQUISA OPERACIONAL E LOGÍSTICA DA MARINHA, 19., 2019, Rio de Janeiro, RJ. Anais [...]. Rio de Janeiro: Centro de Análises de Sistemas Navais, 2019.

\section{INTRODUÇÃO}

Desde os anos 70 o problema de alocação de férias e força de trabalho tem sido um campo amplo para a a realização de pesquisas nas áreas de gestão e pesquisa operacional, tanto em aspectos teóricos quanto práticos [1]. Porém, os problemas tratados na atualidade são bem distintos dos problemas tratados nos estudos pioneiros [2, 3], sendo que essa evolução se dá pela diferenciação ao longo do tempo das necessidades de trabalho, pela consideração das habilidades com a inserção de novas tecnologias e por variações da divisão de turnos.

O problema de alocação de férias e força de trabalho é presente em qualquer organização, seja em uma empresa de pequeno, médio ou grande porte. Estudos sobre o tema são encontrados na literatura em diversos ambientes, como a criação de escalas e divisão de trabalho em call centers [4], transportes aéreos [5], serviços postais [6], serviços de saúde [7], caminhões-tanques [8], entre diversos outros. Revisões completas sobre o tema são apresentadas em [9, 10, 11, 12, 13].

Entretanto, mesmo sendo um problema comum e conhecido, encontrar boas soluções é normalmente uma tarefa difícil dado à sua característica combinatorial, sendo ainda mais difícil encontrar a solucão ótima de forma a minimizar os custos. 
adequar o planejamento às preferências dos trabalhadores, distribuir os turnos de forma igualitária e satisfazer às restrições de necessidade de trabalho [10]. Ainda, esse problema cresce sua complexidade na medida em que o porte da organização aumenta, dado o crescimento no número de funcionários e habilidades requisitas. Sendo assim, este é um problema bem relevante principalmente para empresas de médio e grande porte.

Um outro agravante na resolução do problema está na consideração do conjunto de habilidades existentes na organização. Em planejamento da força de trabalho, a habilidade é a capacidade de um trabalhador de realizar uma determinada atividade [14]. A habilidade afeta diretamente a forma como a atividade é executada e o grau de eficiência durante esta execução (em questões de tempo e qualidade, por exemplo).

Frente aos aspectos e variações do problema de alocação de mão de obra de trabalhadores, alguns estudos discorrem sobre as técnicas de solução utilizadas para sua resolução e as áreas de aplicação deste problema [11, sendo elas tanto industriais quanto em serviços. Entre as técnicas de resolução, destacam-se aquelas na área de pesquisa operacional, como a programação linear, programação inteira mista e métodos heurísticos.

Este artigo trata do problema considerando cenários do setor bancário. A contribuição deste estudo está na apresentação de uma abordagem de suporte à decisão (ASD) para o planejamento da alocação de férias e cobertura da força de trabalho nesse ambiente, removendo assim a subjetividade da decisão empírica. Este artigo é organizado como segue: a Seção 2 faz uma breve descrição do problema, abordando suas restrições e objetivos; a Seção 3 descreve a abordagem de solução proposta; a Seção 4 contextualiza o estudo de caso alvo deste trabalho; a Seção 5 demonstra e discute os resultados obtidos; por fim, a Seção 6 apresenta as considerações finais.

\section{DESCRIÇÃO DO PROBLEMA}

O problema deste estudo consiste em um conjunto de agências bancárias possuindo, cada uma, um conjunto de trabalhadores qualificados que realizam atividades operacionais ou gerenciais. Cada trabalhador tem um prazo no qual suas férias devem ser gozadas e, dependendo de sua função, seu posto deve ser ocupado por outro trabalhador durante o período de concessão. Existe um conjunto de trabalhadores, denominados "volantes", que são responsáveis por realizar as coberturas em todo o conjunto de agências. Para a realização das coberturas, as qualificações do trabalhador e do volante devem ser compatíveis.

Neste problema, cada trabalhador possui períodos de férias semelhantes (30 dias) que devem ser gozados na totalidade e de forma ininterrupta. Todos os trabalhadores devem ter suas férias concedidas no horizonte anual de planejamento.

Para que seja considerada viável, o planejamento de concessões de férias e cobertura deve possuir as seguintes características: 
- O período máximo permitido para a concessão de férias do trabalhador não pode ser excedido.

- O período de férias não pode ser dividido.

- Todas as concessões devem ser realizadas no período anual de planejamento.

- Se a função do trabalhador requer cobertura, esta deve ser realizada por um volante.

- Um volante só pode realizar a cobertura de um trabalhador se suas habilidades forem compatíveis.

- Um volante não pode realizar mais de uma cobertura por vez.

- Um volante não pode realizar cobertura durante o seu próprio período de férias.

Ao mesmo tempo, para a realização de um bom planejamento, este deve ser construído visando os seguintes objetivos:

- Alocar as coberturas a um determinado volante de forma com que a agência do trabalhador a ser coberto seja o mais próximo póssivel da base na qual o volante está alocado.

- Atribuir, em uma mesma agência, o mínimo de concessões necessárias psimultaneamente de modo a evitar grandes flutuações na força de trabalho local.

- Utilizar o mínimo de volantes quanto possível para a realização das coberturas.

\section{ABORDAGEM DE SOLUÇÃO DO PROBLEMA}

Para a resolução do problema proposto neste estudo foi desenvolvido uma abordagem de suporte à decisão (ASD) composto pela integração de métodos heurísticos e dois modelos matemáticos, esses baseados em problemas de designação e sequenciamento de tarefas.

A solução do problema é construída pelo ASD em quatro etapas de decisão. A primeira avalia se os volantes são suficientes para a realização das coberturas dentro do horizonte proposto, adicionando volantes artificiais ao problema em caso de necessidade. Na segunda etapa, um algoritmo de busca avalia os trabalhadores nos quais a necessidade de cobertura devem ser removidas. Já na terceira etapa, um modelo matemático é responsável pela realização da atribuição das coberturas, informando o volante responsável pela cobertura de férias de cada trabalhador, Por fim, outro modelo matemático é utilizado na quarta etapa para a alocação das datas de concessão de férias de cada trabalhador, inclusive dos volantes. 


\subsection{REMOÇÃO DA NECESSIDADE DE COBERTURA}

A etapa apresentada nesta subseção torna-se necessária por uma peculiaridade existente no cenário utilizado no estudo de caso desta pesquisa. Dentre as funções exercidas nas agências de atendimento, as funções "Gerente de Relacionamento" e "Gerente Geral" possuem habilidades semelhantes e, assim, podem realizar a cobertura mútua no caso da existência de ambos em uma mesma agência. Também, caso existam mais de um trabalhador para uma destas funções, a cobertura mútua também pode ser realizada. Uma agência não pode ficar, em nenhum momento do horizonte de planejamento, sem uma das duas habilidades. Um detalhamento de todas as funções existentes no ambiente utilizado é apresentado na Seção 4.

Buscando a redução da necessidade de cobertura por parte do volante responsável por tal função, os períodos de férias para os gerentes que possuem este cenário, em uma mesma agência, devem ser concedidos em momentos distintos, excluindo a necessidade de cobertura destes trabalhadores. O Algoritmo 1 demonstra o processo de análise realizado.

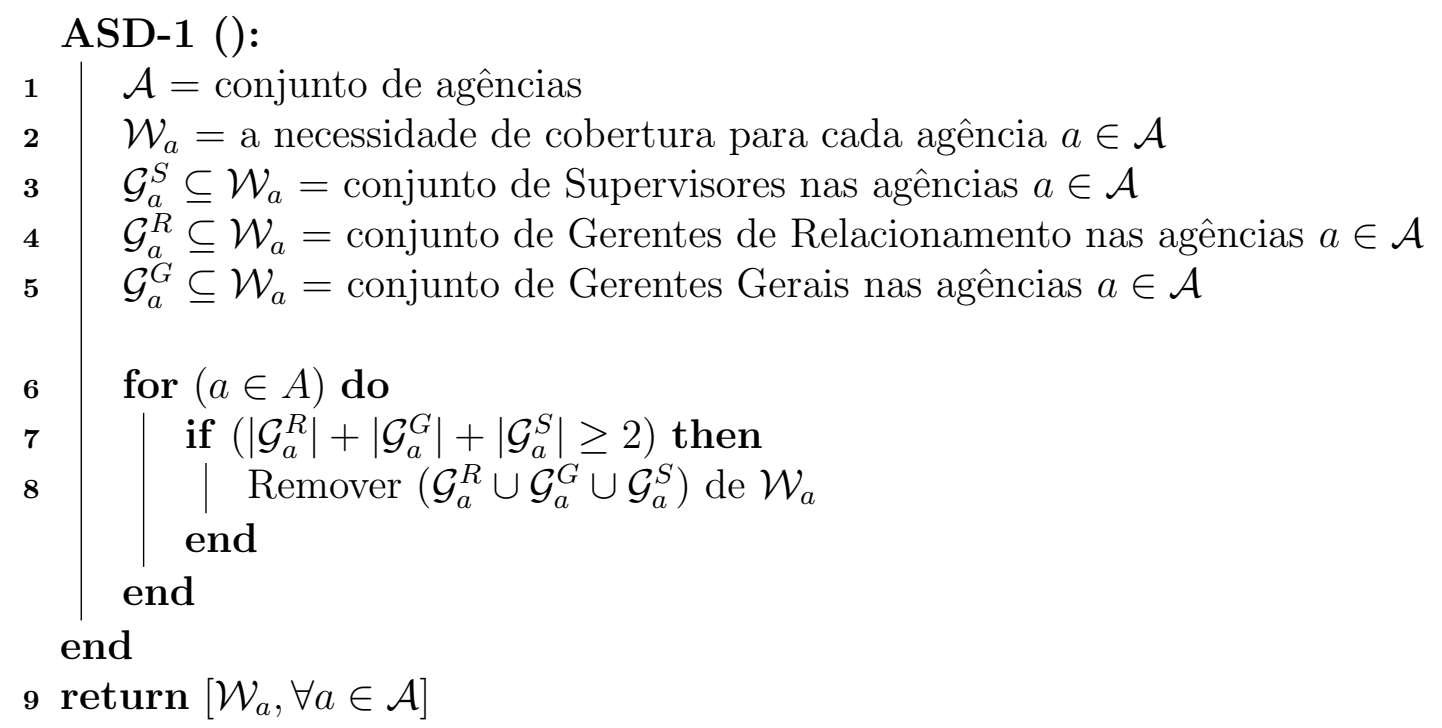

Algoritmo 1: Procedimento para remoção de necessidade de coberturas.

\subsection{AVALIAÇÃO DA VIABILIDADE DE COBERTURA}

De forma a avaliar se existe disponibilidade suficiente de volantes para que se realize as coberturas necessárias, esta etapa tem por objetivo a avaliação da capacidade de cobertura existente. Seja $\mathcal{F}$ o conjunto de funções existentes e $\mathcal{M}_{f}$ os conjuntos de volantes disponíveis para cada função $f \in \mathcal{F}$, a capacidade máxima de cobertura para cada função é dada pela capacidade de cobertura de um volante multiplicado pela quantidade de volantes existentes para a função. 
Caso não exista capacidade suficiente para a demanda existente em uma determinada função $f \in \mathcal{F}$, volantes artificiais devem ser adicionados no conjunto $\mathcal{M}_{f}$ de forma a viabilizar a execução das próximas etapas do ASD. O Algoritmo 2 apresenta a método heurístico responsável por esta análise.

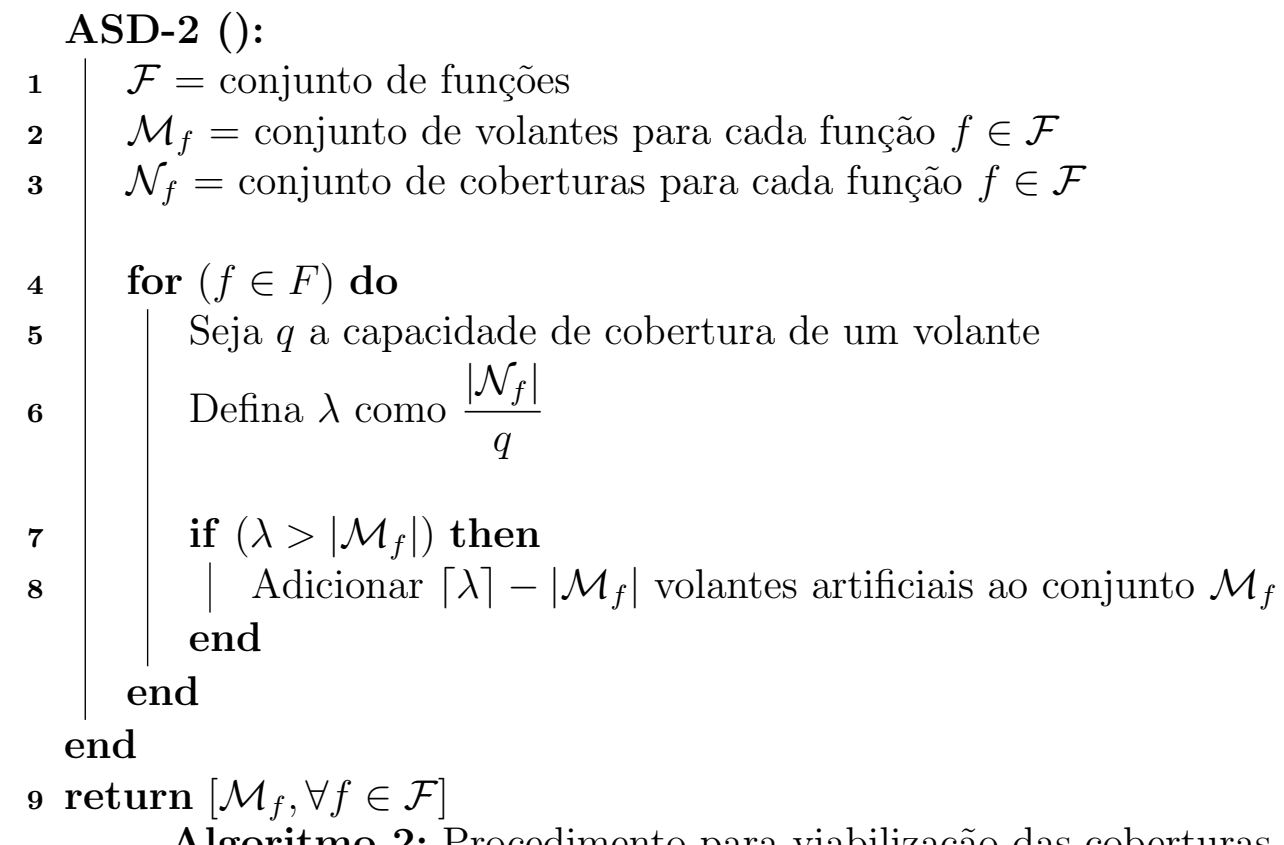

Os volantes artificiais demonstram a necessidade de contratação para que todos os trabalhadores tenham suas concessões realizadas dentro do horizonte de planejamento. De forma complementar, as coberturas atribuídas aos volantes artificiais demonstram os trabalhadores que não poderão usufruir de suas férias caso não ocorram as contratações apontadas.

\subsection{DEFINIÇÃO DA MATRIZ DE COBERTURA}

Para a realização desta etapa, utiliza-se neste trabalho uma execução iterativa do Problema de Designação Generalizada. Neste problema, tem-se $\mathcal{M}$ agentes e $\mathcal{N}$ atividades tal que $|\mathcal{M}|<|\mathcal{N}|$, cada atividade deve ser executada por um único agente e cada agente pode executar mais de uma tarefa. Este problema é dito como clássico na literatura, sendo sua modelagem descrita por [15] e apresentada nas equações (1, 4).

$$
\begin{gathered}
\min \sum_{i \in \mathcal{M}} \sum_{j \in \mathcal{N}} c_{i j} X_{i j} \\
\sum_{i \in \mathcal{M}} X_{i j}=1, \quad \forall j \in \mathcal{N}
\end{gathered}
$$




$$
\begin{gathered}
\sum_{j \in \mathcal{N}} a_{i j} X_{i j}, \leq b_{i}, \quad \forall i \in \mathcal{M} \\
X_{i j} \in\{0,1\}, \quad \forall i \in \mathcal{M} \quad \forall j \in \mathcal{N}
\end{gathered}
$$

A função objetivo (1) minimiza os custos de alocação das atividades aos agentes. As restrições (2) garantem que toda atividade será executada por somente um agente, enquanto as restrições (3) definem que a capacidade $b_{i}$ do agente $i$ não pode ser violada. As restrições (4) informam o domínio das variáveis. Por fim, as variáveis $X_{i j}$ informam se a tarefa $j$ foi designada para o agente $i$.

Para aplicação do modelo de designação no cenário apresentado em nosso estudo, o conjunto $\mathcal{M}$ de agentes é composto pelos volantes, o conjunto $\mathcal{N}$ de atividades é composto pelas necessidades de cobertura, os custos $c_{i j}$ são definidos a partir da distância entre a agência ao qual o volante pertence e a agência no qual será realiza a cobertura. Deste modo, a função objetivo (1) busca a minimização da distância total percorrida por todos os volantes para a realização das coberturas de férias.

Um ponto importante a ser observado durante o processo de alocação das cobertuas é que o número de coberturas realizadas por volantes de mesma função devem ser o mais próximas quanto possível. Para gerar tal equilíbrio no modelo apresentado, o capacidade $b_{i}$ de cada agente foi definida segundo a equação (5). Os parâmetros $a_{i j}$ foram definidos conforme a equação (6). Desta forma, as restrições (3) passam a informar a quantidade máxima de coberturas a serem realizadas pelos agentes.

$$
\begin{gathered}
b_{i}=\frac{|\mathcal{M}|}{|\mathcal{N}|}+1, \quad \forall i \in \mathcal{M} \\
a_{i j}=1, \quad \forall i \in \mathcal{M} \quad \forall j \in \mathcal{N}
\end{gathered}
$$

O modelo é executado de tal forma que, em cada execução, o conjunto $\mathcal{M}$ de volantes e o conjunto $\mathcal{N}$ de necessidades de cobertura sejam definidos para cada função no qual coberturas são requisitadas. O Algoritmo 3 apresenta o procedimento proposto.

\subsection{SEQUENCIAMENTO DAS CONCESSÕES DE FÉRIAS}

Para a atribuição dos momentos de concessão das férias aos trabalhadores foi proposto neste trabalho uma modelagem matemática possuindo premissas de um Job Shop Scheduling. Neste problema, tem-se $\mathcal{N}$ tarefas a serem executadas em $\mathcal{R}$ recursos e por $\mathcal{J}$ operadores, sendo que cada recurso executa somente uma gama determinada de tarefas e as tarefas possuem relação de precedência entre si. As relações de precedência, bem como a alocação de determinadas tarefa em um mesmo 


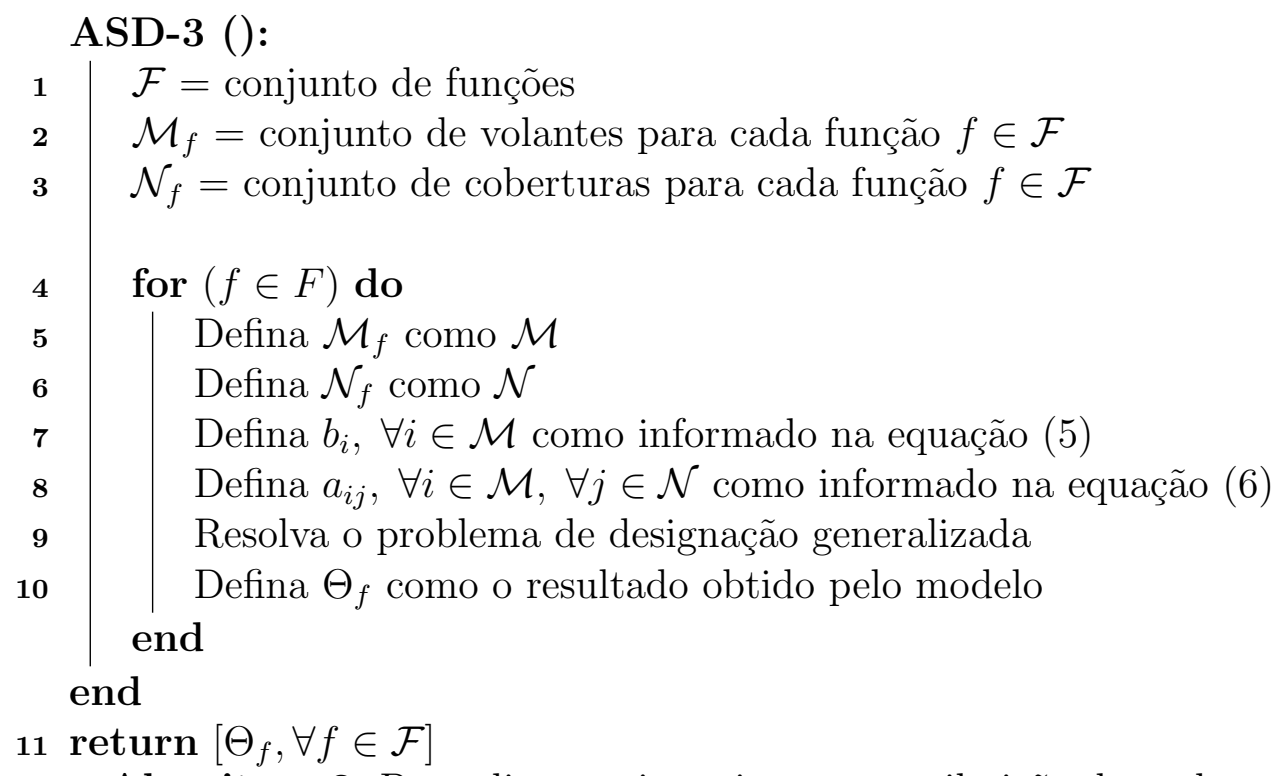

Algoritmo 3: Procedimento iterativo para atribuição das coberturas.

recurso ou operador, fazem com que essas não possam ser executadas paralelamente, devendo uma aguardar o término de outra.

Sendo o tempo de férias a ser cedido ao trabalhador considerado neste modelo como a duração $d_{j}$ da tarefa $j \in \mathcal{N}$, o modelo proposto nas equações $(7$ - 13) busca encontrar uma solução viável para o problema de forma com que todas as tarefas (períodos de férias) sejam alocados dentro do horizonte de planejamento, sendo respeitadas as restrições de não paralelismo.

As restrições (7) e (8) possuem o papel de atribuir os momentos de execução das tarefas. As restrições (9) e (10) informam se duas atividades $i$ e $j$ foram alocadas paralelamente. As restrições (11) garantem que não existirão paralelismos entre atividades que forem executadas em um mesmo recurso ou por um mesmo operador. As restrições (12) delimitam o tempo máximo de término de uma determinada tarefa $j$, de acordo com seu deadline de produção, e o tempo mínimo de início da tarefa, em caso de limitações tecnológicas. Por fim, as variáveis $F_{j}$ informam o momento de término da tarefa $j$, as variáveis $P_{i j}$ informam se existe paralelismo entre duas tarefas $i$ e $j$, sendo $P_{i j}^{1}$ e $P_{i j}^{2}$ variáveis auxiliares para a definição de $P_{i j}$.

$$
\begin{gathered}
F_{i}-F_{j}+d_{j} \leq M \cdot P_{i j}^{1} \quad \forall i \in \mathcal{N}, \forall j \in \mathcal{N}, i \neq j \\
F_{j}-F_{i}+d_{i} \leq M \cdot P_{i j}^{2} \quad \forall i \in \mathcal{N}, \forall j \in \mathcal{N}, i \neq j \\
P_{i j}^{1}+P_{i j}^{2}-1 \leq P_{i j} \quad \forall i \in \mathcal{N}, \forall j \in \mathcal{N}, i \neq j \\
P_{i j}^{1}+P_{i j}^{2} \geq 2 \cdot P_{i j} \quad \forall i \in \mathcal{N}, \quad \forall j \in \mathcal{N}, i \neq j
\end{gathered}
$$




$$
\begin{aligned}
& P_{i j} \leq 0 \quad \forall(i, j) \in\left(\psi^{1} \cup \psi^{2} \cup \psi^{3}\right) \\
& d_{i}^{\text {min }}+d_{i} \leq F_{i} \leq d_{i}^{\text {max }} \quad \forall i \in \mathcal{N} \\
& P_{i j} \in\{0,1\}, \quad \forall i \in \mathcal{N} \quad \forall j \in \mathcal{N} \\
& P_{i j}^{1} \in\{0,1\}, \quad \forall i \in \mathcal{N} \quad \forall j \in \mathcal{N} \\
& P_{i j}^{2} \in\{0,1\}, \quad \forall i \in \mathcal{N} \quad \forall j \in \mathcal{N}
\end{aligned}
$$

Para a aplicação do modelo de sequenciamento de tarefas proposto no cenário apresentado, o conjunto $\mathcal{N}$ de atividades é composto pelas necessidades de cobertura. Os conjuntos $\psi$, que são responsáveis por armazenar as tuplas de paralelismos que não podem ocorrer, são gerados baseados em três decisões: $\psi^{1}$ é formado pelas relações de não paralelismos advindas das decisões de coberturas. Trabalhadores que terão suas coberturas realizadas por um mesmo volante não podem gozar de suas férias simultaneamente. $\psi^{2}$ é formado pelos trabalhadores que se enquadram nos casos em que é possível que coberturas sejam realizadas sem a alocação de um volante. Esses casos são detalhados na Seção 4. $\psi^{3}$ é formado pelas relações de não paralelismo interno à agência de atendimento. Para todas as agências, exceto MTZ, foram definidas tuplas de forma que nenhum paralelismo ocorra. Já para a agência MTZ, que possui quantidade de trabalhares que impossibilita o não paralelismo ao longo do ano, criou-se dois subgrupos de trabalhadores de forma que as tuplas fossem criadas internas a esse subgrupo. Os trabalhadores foram divididos de forma aleatória e igualitária.

\section{ESTUDO DE CASO}

O objeto deste estudo se passa em uma Cooperativa de Crédito do estado de Minas Gerais. A estrutura da organização é composta por uma sede administrativa e outras dezessete agências de atendimento espalhadas pelo estado. O estudo possui foco em seis categorias de funções que são apontadas como críticas pela organização, sendo elas: Gerêntes Gerais (GG), Gerentes de Relacionamento (GR), Agentes de Atendimento (AA), Caixas Tesoureiros (CT) e Supervisores (SV). Para os trabalhadores das funções descritas, têm-se volantes responsáveis pelas coberturas de férias. Ao todo, as funções consideradas neste trabalho somam 92 trabalhadores, sendo eles 15 Gerentes Gerais, 12 Gerentes de Relacionamento, 38 Agentes de Atendimento, 25 Caixas Tesoureiros e 2 Supervisores.

Visando atender a demanda das diferentes habilidades nas diferentes cidades, as agências de atendimento possuem quantidades distintas de trabalhadores para cada funcão. A Tabela 1 apresenta essa distribuicão. 
Tabela 1: Distribuição dos trabalhadores por categoria de função e por agência de atendimento.

\begin{tabular}{lccccc}
\hline Agência & CT & GG & GR & SV & AA \\
\hline MTZ & 4 & 3 & 1 & 0 & 5 \\
RPC & 1 & 0 & 0 & 0 & 3 \\
NVE & 2 & 1 & 0 & 0 & 2 \\
PRT & 2 & 1 & 0 & 0 & 2 \\
CRZ & 3 & 1 & 1 & 1 & 1 \\
BVL & 1 & 0 & 0 & 0 & 1 \\
STB & 1 & 1 & 1 & 0 & 2 \\
BCC & 1 & 0 & 0 & 1 & 3 \\
ALV & 1 & 1 & 0 & 0 & 3 \\
ITA & 3 & 1 & 1 & 0 & 4 \\
BLZ & 0 & 1 & 3 & 0 & 1 \\
OPT & 2 & 0 & 2 & 0 & 0 \\
CAE & 1 & 1 & 0 & 0 & 2 \\
MAR & 1 & 1 & 2 & 0 & 4 \\
ITO & 1 & 1 & 0 & 0 & 2 \\
NVL & 1 & 1 & 1 & 0 & 2 \\
OUB & 0 & 1 & 0 & 0 & 1 \\
\hline
\end{tabular}

Para que as concessões de férias sejam alocadas, a cooperativa de cŕedito possui trabalhadores específicos que realizam a cobertura para as respectivas categorias de funções. Estes trabalhadores serão chamados de "volantes". Desta forma, decisões quanto a alocação da força de trabalho dos volantes devem ser efetivadas para a realização das coberturas. Existem três casos em que as agências de atendimento podem realizar a cobertura de forma interna, ou seja, sem a necessidade da alocação de um volante.

- Caso 1: a agência de atendimento possui as funções GG e GR.

- Caso 2: a agência possui mais de um trabalhador GG ou GR.

- Caso 3: a agência possui a função SV e as funções GG ou GR.

Uma vez que se tenha a ocorrência dos casos anteriores, se a concessão das férias dos trabalhadores envolvidos nas funções informadas não ocorrerem paralelamente, as coberturas por volantes não são necessárias. As funções AA e CT devem, obrigatoriamente, ter suas concessões de férias cobertas por um volante.

A empresa objeto deste estudo não conta com um dimensionamento adequado de volantes, de forma a não saber se sua quantidade é suficiente para o atendimento da demanda de coberturas a serem realizadas no ano. Por este motivo, é comum que contratações emergenciais de volantes ocorram ou que trabalhadores não tenham 
Tabela 2: Número de coberturas realizadas e taxa de ocupação dos volantes reais e artificiais.

\begin{tabular}{lcrr}
\hline Volante & Categoria & Coberturas & Ocupação \\
\hline Volante 1 & CT & 8 & $67 \%$ \\
Volante 2 & CT & 9 & $75 \%$ \\
Volante 3 & CT & 8 & $67 \%$ \\
Volante 4 & GG/GR/SV & 6 & $50 \%$ \\
Volante 5 & AA & 10 & $83 \%$ \\
Artificial 1 & AA & 10 & $83 \%$ \\
Artificial 2 & AA & 10 & $83 \%$ \\
Artificial 3 & AA & 8 & $67 \%$ \\
\hline
\end{tabular}

suas férias liberadas, gerando descontentamentos e acumulando demandas de cobertura para o próximo ano.Atualmente, a Cooperativa de Crédito possui três volantes $\mathrm{CT}$, um volante GG/GR/SV e um volante AA.

Outro fator existente é a descentralização da tomada de decisão sobre as concessões e coberturas, acarretando em paralelismos e reposições que poderiam ser evitadas. No atual método de trabalho da empresa, essa tarefa é realizada tendo como foco o curto prazo, forçando o gestor a revisar o planejamento diversas vezes ao longo do horizonte de planejamento.

\section{RESULTADOS E DISCUSSÕES}

Os experimentos computacionais foram realizados utilizando os dados reais fornecidos pela Cooperativa de Crédito objeto deste estudo. Todos os códigos foram implementados em Python 2.7 e os modelos foram solucionados utilizando o solver comercial Gurobi Optimizer. Os experimentos foram realizados em um notebook com processador Inter(R) Core(TM) i5-4210U 1.70GHz, 8 GB de memória RAM DDR31600 MHz e sistema operacional Ubuntu 16.04.

A ASD proposta foi capaz de solucionar o problema apresentado pela empresa. Para a viabilização das coberturas, a etapa ASD-2 realizou a criação de 3 volantes artificiais do tipo AA. As categorias, quantidades de coberturas realizadas e taxas de ocupação dos volantes reais e artificiais são apresentados na Tabela 2. Para critério de comparação, no último período de planejamento, a empresa conseguiu conceder férias a somente $58 \%$ de sua força de trabalho, ou seja, 53 dos 92 funcionários.

Quanto ao número de concessões, a tomada de decisão de forma centralizada aumentou o nível deste parâmetro ao longo dos meses do ano. A Figura 1 demonstra as quantidades de concessões de férias mensais para a ASD proposta e para a abordagem atual adotada pela empresa e aplicada no último período de planejamento. Em conjunto, o método também se mostrou eficiente ao minimizar a quantidade de concessões paralelas em uma mesma agência de atendimento, ocorrendo paralelismo em somente uma das agências. 


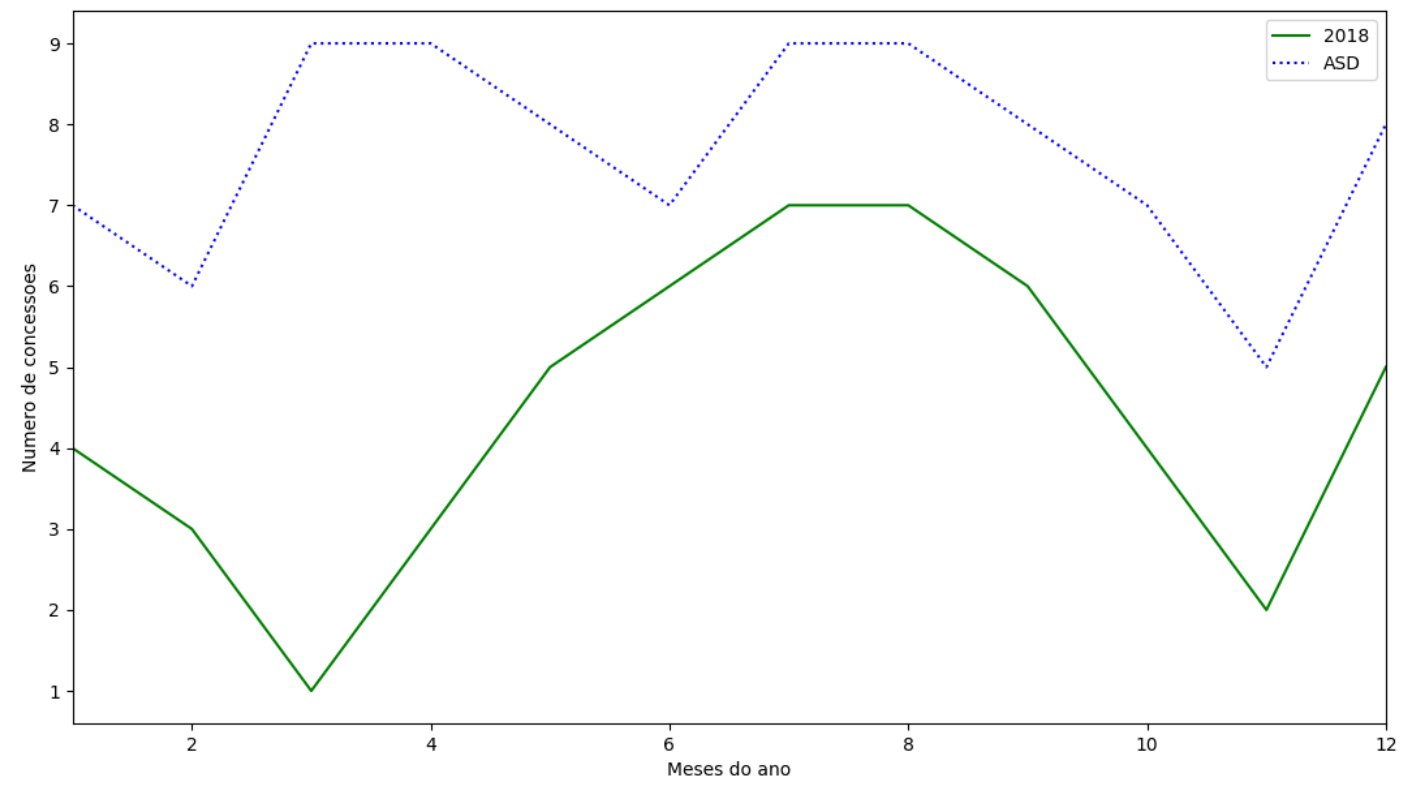

Figura 1: Número de concessões ao longo do período de planejamento.

Observa-se com a criação dos volantes artificiais que a atual capacidade de cobertura não se mostra suficiente para o atendimento das demandas anuais de férias da empresa. Ressalta-se neste momento que a atribuição de volantes artificiais demonstra, em âmbitos práticos, a necessidade de contratação de novos trabalhadores responsáveis por realizar coberturas de férias. Deste modo, os trabalhadores que tiveram suas coberturas atribuídas aos volantes artificais não poderiam, no atual cenário, realizar a solicitação de seu período de férias. Essas demandas seriam alocadas no próximo perído de planejamento, ou seja, no próximo ano.

Outro aspecto a ser observado é a quantidade de coberturas realizadas para a função GG/GR/SV. Foram realizados somente 6 coberturas em um total de 29 trabalhadores da categoria, demonstrando que o método proposto explorou a utilização dos casos em que a cobertura pode acontecer internamente na agência (Tabela 5).

\section{CONSIDERAÇÕES FINAIS}

Esta pesquisa apresenta uma abordagem de otimização baseada em modelos matemáticos e heurísticas para lidar com o planejamento da força de trabalho e concessão de férias, avaliando cenários de alocação para funcionários volantes de múltiplas agências de uma Cooperativa de Crédito. Os resultados foram discutidos e validados pelos gerentes da empresa colaboradora desta pesquisa.

Os resultados computacionais demonstraram que as soluções são viáveis de serem colocadas em prática. Por se tratar de um sistema automatizado de apoio a decisão, a abordagem proposta gera soluções em tempos inferiores aos praticados atualmente 
pela empresa, gerando grande potencial de redução de esforços operacionais.

Para pesquisas futuras, os autores deste trabalho apontam a inserção de aspectos existentes nas legislações nacionais para concessão de férias, como limitações nas datas de início, por exemplo. Sugere-se também a consideração da logística de cobertura, de tal forma que uma vez que um volante vá para uma deterinada agência, ele permaneça nela até que todas as coberturas alocadas a ele naquela mesma agência sejam aplicadas. Este estudo permanece na agenda dos autores.

\section{REFERÊNCIAS BIBLIOGRÁFICAS}

[1] COSTA, M.-C.; JARRAY, F.; PICOULEAU, C. An acyclic days-off scheduling problem. 4OR, Springer, v. 4, n. 1, p. 73-85, 2006. 2

[2] DANTZIG, G. B. Letter to the editor - a comment on edie's "traffic delays at toll booths". Journal of the Operations Research Society of America, INFORMS, v. 2 , n. 3 , p. 339-341, 1954. 2

[3] EDIE, L. C. Traffic delays at toll booths. Journal of the operations research society of America, INFORMS, v. 2, n. 2, p. 107-138, 1954. 2

[4] BHULAI, S.; KOOLE, G.; POT, A. Simple methods for shift scheduling in multiskill call centers. Manufacturing $\&$ Service Operations Management, INFORMS, v. 10, n. 3 , p. $411-420,2008.2$

[5] KOHL, N.; KARISCH, S. E. Airline crew rostering: Problem types, modeling, and optimization. Annals of Operations Research, Springer, v. 127, n. 1-4, p. 223-257, 2004. 2

[6] BARD, J. F.; BINICI, C. et al. Staff scheduling at the united states postal service. Computers \& Operations Research, Elsevier, v. 30, n. 5, p. 745-771, 2003. 2

[7] BURKE, E. K. et al. The state of the art of nurse rostering. Journal of scheduling, Kluwer Academic Publishers, v. 7, n. 6, p. 441-499, 2004. 2

[8] SOLOS, I. P.; TASSOPOUlOS, I. X.; BELIGIANNIS, G. N. Optimizing shift scheduling for tank trucks using an effective stochastic variable neighbourhood approach. Int. J. Artif. Intell, v. 14, n. 1, p. 1-26, 2016. 2

[9] ERNST, A. T. et al. An annotated bibliography of personnel scheduling and rostering. Annals of Operations Research, Springer, v. 127, n. 1-4, p. 21-144, 2004. 2 
[10] ERNST, A. T. et al. Staff scheduling and rostering: A review of applications, methods and models. European journal of operational research, Elsevier, v. 153, n. 1, p. 3-27, 2004. 2, 3

[11] BRUCKER, P.; QU, R.; BURKE, E. Personnel scheduling: Models and complexity. European Journal of Operational Research, Elsevier, v. 210, n. 3, p. 467-473, 2011. 2, 3

[12] BERGH, J. Van den et al. Personnel scheduling: A literature review. European journal of operational research, Elsevier, v. 226, n. 3, p. 367-385, 2013. 2

[13] BRUECKER, P. D. et al. Workforce planning incorporating skills: State of the art. European Journal of Operational Research, Elsevier, v. 243, n. 1, p. 1-16, 2015. 2

[14] SADEGHI-DASTAKI, M.; AFRAZEH, A. A two-stage skilled manpower planning model with demand uncertainty. International Journal of Intelligent Computing and Cybernetics, Emerald Publishing Limited, v. 11, n. 4, p. 526-551, 2018. 3

[15] ARENALES, M. et al. Pesquisa operacional: para cursos de engenharia. [S.l.]: Elsevier Brasil, 2015. 6 
Tabela 3: Momentos de concessões e cobertura de férias da agência ITA por período.

\begin{tabular}{lccll}
\hline Período & Função & Cobertura & Início & Fim \\
\hline MAR & AA & Artificial 2 & $02 / 03 / 19$ & $31 / 03 / 19$ \\
ABR & CT & Volante 2 & $01 / 04 / 19$ & $30 / 04 / 19$ \\
MAI & GR & - & $01 / 05 / 19$ & $30 / 05 / 19$ \\
JUN & CT & Volante 2 & $31 / 05 / 19$ & $29 / 06 / 19$ \\
AGO & AA & Artificial 2 & $04 / 07 / 19$ & $02 / 08 / 19$ \\
SET & GG & - & $03 / 08 / 19$ & $01 / 09 / 19$ \\
OUT & CT & Volante 2 & $02 / 09 / 19$ & $01 / 10 / 19$ \\
NOV & AA & Artificial 2 & $01 / 11 / 19$ & $30 / 11 / 19$ \\
DEZ & AA & Artificial 2 & $01 / 12 / 19$ & $30 / 12 / 19$ \\
\hline
\end{tabular}

Tabela 4: Agências de atendimento e momentos de cobertura de férias realizados pelo Volante 1.

\begin{tabular}{lll}
\hline Agência & Início & Fim \\
\hline MTZ & $01 / 01 / 19$ & $30 / 01 / 19$ \\
NVE & $31 / 01 / 19$ & $01 / 03 / 19$ \\
MTZ & $06 / 03 / 19$ & $04 / 04 / 19$ \\
PRT & $05 / 04 / 19$ & $04 / 05 / 19$ \\
PRT & $05 / 05 / 19$ & $03 / 06 / 19$ \\
MTZ & $05 / 07 / 19$ & $03 / 08 / 19$ \\
MTZ & $04 / 08 / 19$ & $02 / 09 / 19$ \\
NVE & $01 / 12 / 19$ & $30 / 12 / 19$ \\
\hline
\end{tabular}


Tabela 5: Momentos de concessões e cobertura de férias das funções de gerência e supervisão.

\begin{tabular}{|c|c|c|c|c|}
\hline Agência & Função & Cobertura & Início & Fim \\
\hline ALV & GG & Volante 4 & $01 / 05 / 19$ & $30 / 05 / 19$ \\
\hline $\mathrm{BCC}$ & SV & - & $29 / 08 / 19$ & $27 / 09 / 19$ \\
\hline BLZ & GG & - & 01/04/19 & $30 / 04 / 19$ \\
\hline BLZ & GR & - & 01/12/19 & $30 / 12 / 19$ \\
\hline BLZ & GR & - & 02/03/19 & $31 / 03 / 19$ \\
\hline BLZ & GR & - & 01/01/19 & $30 / 01 / 19$ \\
\hline $\mathrm{CAE}$ & GG & Volante 4 & 02/09/19 & $01 / 10 / 19$ \\
\hline CRZ & GG & - & $02 / 10 / 19$ & $31 / 10 / 19$ \\
\hline CRZ & GR & - & 02/09/19 & $01 / 10 / 19$ \\
\hline CRZ & SV & - & $05 / 05 / 19$ & $03 / 06 / 19$ \\
\hline ITA & GG & - & 03/08/19 & 01/09/19 \\
\hline ITA & GR & - & 01/05/19 & $30 / 05 / 19$ \\
\hline ITO & GG & Volante 4 & 01/04/19 & $30 / 04 / 19$ \\
\hline MAR & GG & - & 04/07/19 & 02/08/19 \\
\hline MAR & GR & - & $01 / 12 / 19$ & $30 / 12 / 19$ \\
\hline MAR & GR & - & 01/11/19 & $30 / 11 / 19$ \\
\hline MTZ & GG & - & 05/04/19 & $04 / 05 / 19$ \\
\hline MTZ & GG & - & 04/06/19 & 03/07/19 \\
\hline MTZ & GG & - & 03/08/19 & $01 / 09 / 19$ \\
\hline MTZ & GR & - & $31 / 01 / 19$ & $01 / 03 / 19$ \\
\hline NVE & GG & Volante 4 & 02/03/19 & $31 / 03 / 19$ \\
\hline NVL & GG & - & $01 / 12 / 19$ & $30 / 12 / 19$ \\
\hline NVL & GR & - & 04/07/19 & 02/08/19 \\
\hline OPT & GR & - & 03/08/19 & 01/09/19 \\
\hline $\mathrm{OPT}$ & GR & - & 02/09/19 & $01 / 10 / 19$ \\
\hline OUB & GG & Volante 4 & 01/12/19 & $30 / 12 / 19$ \\
\hline PRT & GG & Volante 4 & $02 / 10 / 19$ & $31 / 10 / 19$ \\
\hline STB & GG & - & 03/08/19 & 01/09/19 \\
\hline STB & GR & - & 04/07/19 & 02/08/19 \\
\hline
\end{tabular}

